\title{
Produção de diferentes variedades de mandioca em sistema agroecológico
}

\author{
Alineaurea F. Silva ${ }^{1}$, Luiz M. de Santana ${ }^{2}$, Carla R. R. S. França ${ }^{3}$, Clésio A. de S. Magalhães ${ }^{1}$, \\ Cândido R. de Araújo ${ }^{1}$ \& Sérgio G. de Azevedo ${ }^{1}$
}

\begin{abstract}
RESUMO
Neste trabalho, conduzido na comunidade de Morro Alto, município de Acauã, PI, localizada na Serra da Parreira, situada a $620 \mathrm{~m}$ de altitude, objetivou-se avaliar o crescimento e a produção da mandioca em cultivo consorciado com guandu, sorgo, caupi, algodão e girassol, em sistema agroecológico de produção. A altura, retenção foliar e sobrevivência das plantas de mandioca, não apresentaram diferenças significativas, considerando-se os tipos de consórcio. Observou-se diferença apenas entre variedades na sobrevivência das plantas, sendo que a variedade Do Céu (BGM 537) apresentou a maior porcentagem de sobrevivência. As espécies consorciadas mantiveram diferentes teores de umidade do solo destacando-se o guandu como a cultura onde se encontrou maior teor de umidade do solo. Por outro lado, a produção por hectare variou de 13 a $26 \mathrm{t}$, dependendo da variedade utilizada. Esta produtividade, considerada acima da média da região $\left(12 \mathrm{t} \mathrm{ha}^{-1}\right)$, mostra que o cultivo consorciado, principalmente com leguminosas como o feijão caupi e guandu, pode melhorar não só o rendimento da cultura mas também as características do solo, em áreas dependentes de chuva.
\end{abstract}

Palavras-chave: agricultura de sequeiro, calagem, consórcio, forragem da parte aérea

\section{Growth and production of different cassava varieties in an agroeological system}

\begin{abstract}
This study was conducted in the Morro Alto community, municipal district of Acauã (PI), located in Serra da Parreira, $620 \mathrm{~m}$ altitude. The objective was to evaluate the growth and production of the cassava in the intercropping with guandu, sorgo, caupi, cotton and sunflower in an agroecological production system. The height, foliar retention and survival of the cassava plants did not present significant differences considering the consortium types. A difference was only observed among varieties in relation to the survival of the plants, the variety "Do Céu" (BGM 537) showing the greatest survival percentage. The species intercropping maintained different soil humidity contents, guandu standing out as the crop that maintained the largest soil humidity content $(10 \%)$, even so the consortium type did not modify the productivity of roots. It was observed that the production of roots varied from 13 to $26 \mathrm{tha}^{-1}$, depending on the variety used. This productivity, considered above average for the region $\left(12 \mathrm{t} \mathrm{ha}^{-1}\right)$, shows that intercropping, mainly with legumes such as the bean caupi and guandu, can improve not only farmer's revenue but also soil characteristics in rainfed areas.
\end{abstract}

Keywords: rainfed agriculture, liming of soil, consortium, shoot forage

1 Embrapa Semi-Árido. BR 428, KM 152, Zona Rural, CP 23. CEP 56302-970, Petrolina, PE. Fone: (87) 3862-1711, Fax: (87) 3862-1744. E-mail: alinefs@cpatsa.embrapa.br; clesiomagalhaes@hotmail.com; candero@cpatsa.embrapa.br; sergio@ cpatsa. embrapa.br

2 CODEVASF 3a SR, Rua Presidente Dutra, 160, CEP 56300-000, Petrolina, PE. Fone: (87) 3866-7700, Fax: (87) 3866-7700. E-mail: luiz.manoel@codevasf.gov.br; 3 UFRPE, Pró-Reitoria de Pesquisa e Pós-Graduação, Rua Dom Manoel de Medeiros, s/n, Bairro Dois Irmãos, CEP 52171-900, Recife, PE. E-mail: carlaregina@yahoo.com.br 


\section{INTRODUÇÃO}

A mandioca (Manihot esculenta Cranz) é uma das principais fontes de carboidratos em diversos países no mundo, cuja área plantada é uma das mais representativas dentre as culturas amiláceas. A capacidade de usar água eficientemente, permite sua exploração em regiões de estações secas prolongadas nas quais a cultura ocupa papel predominante nos sistemas de produção agrícola.

Por ser facilmente cultivada e não apresentar grandes exigências nutricionais ou de manejo, a forma de cultivo da planta não difere daquela praticada pelos índios, na época do descobrimento do Brasil (Perpétuo, 2006).

A escolha de variedades mais adaptadas a uma região permite incremento em produtividade da cultura (Fukuda et al., 2003). Muitos agricultores, não sabendo deste fato, insistem em utilizar o mesmo material de plantio que já usavam seus avós, tornando o cultivo pouco produtivo e susceptível a pragas e doenças oportunistas.

O aperfeiçoamento do sistema de produção e o uso de novas tecnologias em cultivos já estabelecidos de mandioca, podem tornar-se uma realidade e melhorar o desempenho dos sistemas produtivos, evitando-se o desmatamento de novas áreas (Melo et al., 2005). Os sistemas agroecológicos prevêem sustentabilidade em longo prazo, o que pode ser conseguido com uma série de práticas ajustadas para cada sistema, principalmente as relacionadas ao solo.

A mandioca é uma cultura que explora o solo com grande intensidade, considerada uma das mais erosivas quando comparada a outras de ciclo curto, como sorgo, milho e amendoim, principalmente nos primeiros meses após o plantio, quando a cobertura do solo ainda é bastante incipiente (Putthacharoen et al., 1998).

Durante seu ciclo, a mandioca não apresenta alta percentagem de cobertura no solo; portanto, sempre que possível, deve ser plantada em consórcio com outras espécies (Otsubo \& Lorenzi, 2004). Muitas espécies de plantas, sobretudo as leguminosas, podem incrementar os teores de matéria orgânica no solo e permitir a fertilização natural de culturas associadas (Moretto et al., 2001; Perin et al., 2002; Ribas et al., 2003). Essas práticas reduziriam o risco de erosão laminar, permitiriam o aporte de nutrientes provenientes da roçagem da cultura intercalar e favoreceriam a retenção de umidade do solo, condição esta altamente desejável para o Semi-Árido Nordestino.

A cobertura vegetal produzida por plantas intercaladas, como o guandu, quando deixada sobre o solo permite uma redução na evaporação da água do solo mantendo, por mais tempo o teor de umidade, além de fornecer, de maneira gradual, os nutrientes contidos em suas folhas. Além do guandu, outras espécies, como a crotalária, gliricídia ou caupi, podem ser utilizadas como adubação verde em cultivo intercalar (Ribas et al., 2003; Lopes et al., 2005), favorecendo o fornecimento de nutrientes e minimizando a exportação de $\mathrm{N}$.

Assim como em quaisquer outros sistemas de cultivo, nos sistemas agroecológicos ou em transição, é preciso que haja análise e comparação para avaliação dos resultados. A com- paração entre diferentes formas de manejo tem, como objetivo, avaliar onde se pode melhorar o sistema e, para tanto, se utilizam os indicadores de sustentabilidade.

Existem diversos tipos de indicadores; os de cultivo, de solo, indicadores sociais etc. (Altieri \& Nichols, 2004). Os indicadores de solo podem ser relacionados aos aspectos químicos, físicos ou biológicos e, além desses, podem ser utilizados indicadores da cultura principal para se avaliar a sustentabilidade dos sistemas em consórcio, proporcionando melhor entendimento dos processos e adoção das práticas por parte dos agricultores.

Propôs-se, neste estudo, avaliar o crescimento e a produção da mandioca para consumo humano e animal, em cultivo consorciado com guandu, sorgo, caupi, algodão e girassol em sistema agroecológico de produção.

\section{MATERIAL E MÉTODOS}

O trabalho foi desenvolvido no município de Acauã, PI, na comunidade de Morro Alto, Serra da Parreira, localizada na divisa dos estados de Pernambuco e Piauí. A comunidade Morro Alto está estabelecida em uma região com clima tropical quente e seco; apresenta período chuvoso de novembro a março e possui as seguintes coordenadas geográficas: $08,21^{\circ}$ de latitude Sul, $41,08^{\circ}$ de longitude Oeste e $620 \mathrm{~m}$ de altitude. Antes da implantação do trabalho, houve várias reuniões com agricultores da localidade, com apoio da Associação dos Pequenos Agricultores do Morro Alto, o que permitiu se obter um breve diagnóstico sobre as aptidões e aspirações dos agricultores locais.

Nas reuniões foram discutidos vários aspectos relacionados às atividades produtivas dos agricultores e aos problemas que estavam prejudicando a atividade mandioqueira na região. Algumas alternativas foram levantadas a fim de melhorar a situação, oportunidade em que se sugeriu a implantação de Unidades de Observação com mandioca em sistema produtivo mais rentável e diversificado; fez-se, então, o planejamento das atividades com a participação de 14 famílias de agricultores envolvidos neste trabalho e participantes da Associação dos Pequenos Produtores do Morro Alto. Todo o trabalho contou com ajuda mútua, em parceria entre Associação, Embrapa Semi-Árido, Ministério do Desenvolvimento Agrário (MDA), Ministério do Desenvolvimento Social (MDS) e Prefeitura do Município de Acauã, PI.

Escolheu-se uma área de trabalho coletiva no topo do morro, que perfazia uma pequena parcela do total, mantendo-se ao redor a mata existente e desmatando apenas as áreas necessárias para a instalação das parcelas experimentais. Após o desmatamento monitorado, realizou-se a aração e a gradagem do solo. O mapa da área, com a localização dos campos de cada agricultor familiar e da Unidade de Observação da Embrapa Semi-Árido, foi planejado de maneira participativa nas reuniões periódicas.

Realizou-se, antes de qualquer interferência de ordem agronômica, a amostragem do solo para efetuar a análise físicoquímica (Tabela 1). O solo foi classificado texturalmente 
como franco arenoso, apresentando 69,33\% de areia, 10,33\% de silte e $20,33 \%$ de argila. Os resultados da análise de solo (M. O. 21,77 g dm${ }^{-3}$, $\mathrm{pH}\left(\mathrm{H}_{2} \mathrm{O}-1: 2,5\right)$ 4,7, C.E. $0,18 \mathrm{dS} \mathrm{m}^{-1}$, $\mathrm{P} 2,0 \mathrm{mg} \mathrm{dm}^{-3}, \mathrm{~K}+0,28 \mathrm{cmol}_{\mathrm{C}} \mathrm{m}^{-3}, \mathrm{Ca}^{2+} 0,77 \mathrm{cmol}_{\mathrm{C}} \mathrm{dm}^{-3}, \mathrm{Mg}^{2+}$ $0,73 \mathrm{cmol}_{\mathrm{C}} \mathrm{dm}^{-3}, \mathrm{Na}^{+} 0,02 \mathrm{cmol}_{\mathrm{C}} \mathrm{dm}^{-3}, \mathrm{Al}^{3+} 0,58 \mathrm{cmol}_{\mathrm{C}} \mathrm{dm}^{-3}$,

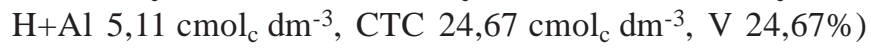
mostraram a necessidade de calagem e, deste modo, se recomendou a adição de 1,5 t ha-1 de calcário dolomítico, visando elevar o seu $\mathrm{pH}$.

O plantio das áreas ocorreu no final do ano de 2004, com a colaboração de todos os agricultores envolvidos no processo. Neste período foram escolhidas as espécies e cultivares a serem utilizadas no sistema de produção. As cultivares de mandioca escolhidas, foram: Engana Ladrão (BGM 1269), Brasília (TSA 128), Do Céu (BGM 537) e Macaxeira Preta (variedade local) consorciadas com Feijão de Corda (Vigna unguiculata), Sorgo Granífero (Sorghum bicolor L. Moench), Feijão Guandu (Cajanus cajan L.), Girassol (Helianthus annus) e Algodão (Gossypium hirsutum L.). O ensaio foi dividido em blocos no fatorial 4 x 5 com 3 repetições; cada parcela experimental foi formada com duas linhas de plantas de mandioca, no espaçamento $1,0 \times 0,8 \mathrm{~m}$, consorciadas de cada lado com duas linhas das culturas anteriormente citadas. Todos os tratos culturais foram realizados pelos agricultores, que obedeceram ao costume local.

Após o período das chuvas, em 2005, iniciou-se a colheita de cada um dos cultivos consorciados, de acordo com a sua maturação. Em julho do mesmo ano, fez-se a primeira avaliação da cultura de mandioca, com a ajuda dos produtores, anotando-se as seguintes características das plantas; altura total da planta (cm), retenção foliar (\%) e sobrevivência (\%). Em fevereiro de 2006 foi a vez do segundo plantio das culturas consorciadas, para manter a cobertura do solo, porém não apresentaram bom desenvolvimento devido ao sombreamento da mandioca e deficiência hídrica do solo.

Em julho de 2006 realizou-se a colheita final da mandioca, coletando-se 3 plantas por parcela e as características avaliadas foram: altura das plantas (cm), a retenção foliar (\%), a sobrevivência das plantas (\%), o peso total da parte aérea (kg), o peso da parte aérea destinada a forragem (kg de ramos mais tenros e com presença de folhas), o peso total de raízes $(\mathrm{kg})$, o número total raízes, o peso da cepa $(\mathrm{kg})$, a produtividade de raiz $\left(\mathrm{kg} \mathrm{ha}^{-1}\right)$, a produtividade de parte aérea $\left(\mathrm{kg} \mathrm{ha}^{-1}\right)$, o peso total da planta $(\mathrm{kg})$, o teor de matéria seca nas raízes (\%) calculado pelo método da gravidade específica (Toro \& Cañas, 2002), a preferência dos agricultores pelas variedades, estimada através de notas dadas no momento da colheita, o índice de colheita (\%) calculado dividindo-se o peso de raízes $(\mathrm{kg})$ pelo peso da planta inteira (kg) e índice de produção de forragem (\%) calculado dividindo-se o material destinado a forragem $(\mathrm{kg})$ pelo material total da parte aérea da planta $(\mathrm{kg})$. No momento da colheita também foram coletadas amostras do solo na profundidade de 0-30 cm nas diferentes parcelas para avaliação do teor de umidade e demais características químicas. Os resultados foram analisados estatisticamente e as médias testadas pelo teste de Tukey, a 5\% de probabilidade.

\section{RESULTADOS E DISCUSSÃO}

Verificou-se a presença de boa quantidade de argila e silte denotando que o solo teria boa retenção de umidade nas condições em que o trabalho foi realizado (Tabela 1). Não houve efeito da aplicação do calcário sobre o pH do solo, o que se deve, provavelmente, à má aplicação do corretivo. A variedade de mandioca local, Macaxeira Preta, apresentou maior capacidade de extrair, do solo, o fósforo e o cálcio, mostrando ser uma variedade exigente em nutrientes. Apesar do manejo de solo adotado no experimento ter elevado os valores da Capacidade de Troca Catiônica (Tabela 1), as outras características químicas do solo se mantiveram constantes e não modificaram o comportamento das plantas nos primeiros seis meses após o plantio (Tabela 2).

Tabela 1. Características químicas e físicas do solo coletado antes da instalação do experimento e após a coleta das plantas de mandioca

\begin{tabular}{lccccc}
\hline Característica & Solo inicial & $\begin{array}{c}\text { Engana } \\
\text { Ladrão }\end{array}$ & Brasília & Do Céu & $\begin{array}{c}\text { Macaxeira } \\
\text { Preta }\end{array}$ \\
M. 0. $\left(\mathrm{g} \mathrm{dm}^{-3}\right)$ & 21,77 & $22,18 \mathrm{~A}^{*}$ & $21,21 \mathrm{~A}$ & $21,31 \mathrm{~A}$ & $18,93 \mathrm{~A}$ \\
$\mathrm{pH}\left(\mathrm{H}_{2} \mathrm{O}-1: 2,5\right)$ & 4,7 & $4,54 \mathrm{~A}$ & $4,55 \mathrm{~A}$ & $4,54 \mathrm{~A}$ & $4,54 \mathrm{~A}$ \\
$\mathrm{C.} \mathrm{E.}\left(\mathrm{dS} \mathrm{m}^{-1}\right)$ & 0,18 & $0,33 \mathrm{~A}$ & $0,32 \mathrm{~A}$ & $0,28 \mathrm{~A}$ & $0,27 \mathrm{~A}$ \\
$\mathrm{P}\left(\mathrm{mg} \mathrm{dm}^{-3}\right)$ & 2,00 & $1,87 \mathrm{AB}$ & $1,97 \mathrm{~A}$ & $1,60 \mathrm{BC}$ & $1,53 \mathrm{C}$ \\
$\mathrm{K}^{+}\left(\mathrm{cmol}_{\mathrm{c}} \mathrm{dm}^{-3}\right)$ & 0,28 & $0,14 \mathrm{~A}$ & $0,13 \mathrm{~A}$ & $0,13 \mathrm{~A}$ & $0,13 \mathrm{~A}$ \\
$\mathrm{Ca}^{2+}\left(\mathrm{cmol}_{\mathrm{c}} \mathrm{dm}^{-3}\right)$ & 0,77 & $1,70 \mathrm{~A}$ & $1,55 \mathrm{~B}$ & $1,46 \mathrm{~B}$ & $1,44 \mathrm{~B}$ \\
$\mathrm{Mg}^{2+}\left(\mathrm{cmol}_{\mathrm{c}} \mathrm{dm}^{-3}\right)$ & 0,73 & $0,65 \mathrm{~A}$ & $0,62 \mathrm{~A}$ & $0,62 \mathrm{~A}$ & $0,64 \mathrm{~A}$ \\
$\mathrm{Na}^{+}\left(\mathrm{cmol}_{\mathrm{c}} \mathrm{dm}^{-3}\right)$ & 0,02 & $0,014 \mathrm{~A}$ & $0,015 \mathrm{~A}$ & $0,012 \mathrm{~A}$ & $0,012 \mathrm{~A}$ \\
$\mathrm{Al}^{3+}\left(\mathrm{cmol}_{\mathrm{c}} \mathrm{dm}^{-3}\right)$ & 0,58 & $0,73 \mathrm{~A}$ & $0,77 \mathrm{~A}$ & $0,77 \mathrm{~A}$ & $0,78 \mathrm{~A}$ \\
$\mathrm{H}+\mathrm{Al}\left(\mathrm{cmol}_{\mathrm{c}} \mathrm{dm}^{-3}\right)$ & 5,11 & $5,70 \mathrm{~A}$ & $5,44 \mathrm{~A}$ & $5,26 \mathrm{~A}$ & $5,27 \mathrm{~A}$ \\
$\mathrm{CTC}\left(\mathrm{cmol}_{\mathrm{c}} \mathrm{dm}^{-3}\right)$ & 6,91 & $8,18 \mathrm{~A}$ & $7,94 \mathrm{AB}$ & $7,48 \mathrm{~B}$ & $7,47 \mathrm{~B}$ \\
$\mathrm{~V}(\%)$ & 24,67 & $29,97 \mathrm{~A}$ & $29,17 \mathrm{~A}$ & $30,20 \mathrm{~A}$ & $29,47 \mathrm{~A}$ \\
\hline
\end{tabular}

* Linhas seguidas de mesma letra não diferem entre si a nível de $5 \%$ de probabilidade, pelo teste Tukey

Após seis meses do plantio não ocorreu interação entre as variedades de mandioca e as espécies consorciadas. As variedades de mandioca não indicaram diferenças significativas quanto às características de crescimento e retenção foliar porém se observou diferença entre as variedades em relação à sobrevivência das plantas, sendo que a variedade Do Céu apresentou maior percentual de sobrevivência (Tabela 2).

Tabela 2. Características da mandioca, seis meses após o plantio

\begin{tabular}{lcrc}
\hline Variedade & $\begin{array}{c}\text { Altura } \\
\text { (cm) }\end{array}$ & $\begin{array}{c}\text { Retençãa } \\
\text { Foliar (\%) }\end{array}$ & $\begin{array}{c}\text { Sobrevivência } \\
\text { (\%) }\end{array}$ \\
Engana Ladrão (BGM 1269) & $46,96 \mathrm{~A}^{*}$ & $27,73 \mathrm{~A}$ & $87,34 \mathrm{~B}$ \\
Brasilia (TSA 128) & $43,82 \mathrm{~A}$ & $25,16 \mathrm{~A}$ & $85,34 \mathrm{~B}$ \\
Do Céu (BGM 537) & $48,53 \mathrm{~A}$ & $31,49 \mathrm{~A}$ & $97,34 \mathrm{~A}$ \\
Macaxeira Preta (variedade local) & $46,83 \mathrm{~A}$ & $31,60 \mathrm{~A}$ & $93,34 \mathrm{AB}$ \\
\hline
\end{tabular}

* Colunas seguidas de mesma letra não diferem entre si a nível de $5 \%$ de probabilidade, pelo teste Tukey

As espécies utilizadas em consórcio com a mandioca, eram plantas de ciclo curto, colhidas no primeiro ano, deixando-se maior espaço para continuidade do crescimento das plantas de mandioca. O sorgo mostrou-se pouco adaptado à condição edafoclimática da região, não apresentando 
produção de grãos nem, tampouco, de material vegetativo (folhas e colmos). A maioria das plantas desta espécie não se estabeleceu em campo, mesmo após o replantio; o algodão também não apresentou produção de capulhos significativa, não sendo considerado para efeito comparativo com as demais espécies. O feijão guandu, o girassol e o feijão caupi, produziram satisfatoriamente (Tabela 3). O feijão caupi foi o que obteve maior produtividade (14,0 g por planta) quando comparado com os demais; esta produção se refere à metade da área da parcela, pois a outra parte ainda permaneceu ocupada com a mandioca que seria colhida no ano seguinte. Existe, portanto, a possibilidade de se adquirir renda com a venda ou eliminação da compra dos grãos do caupi para consumo familiar enquanto se aguarda a colheita da mandioca, ao mesmo tempo em que se melhora a qualidade do solo, por se tratar de planta leguminosa.

Avaliando-se a umidade do solo (Tabela 3) se observa, no período seco, que a utilização de espécies em consórcio, principalmente do feijão guandu, permite manter uma condição de umidade favorável ao desenvolvimento das plantas, por mais tempo.

Tabela 3. Produção de grãos das culturas consorciadas na parcela e umidade do solo no local dessas culturas

\begin{tabular}{lcc}
\hline Espécie Consorciada & $\begin{array}{c}\text { Produção por } \\
\text { parcela 20 } \mathbf{~ m}^{2} \mathbf{~ ( g )}\end{array}$ & $\begin{array}{c}\text { Umidade do solo } \\
\text { no local (\%) }\end{array}$ \\
Feijão caupi (Vigna unguiculata) & 282,75 & $4,01 \mathrm{C}$ \\
Guandu de mesa (Cajanus cajan L.) & 118,63 & $10,92 \mathrm{~A}$ \\
Girassol (Helianthus annus) & 21,25 & $3,74 \mathrm{C}$ \\
Algodão (Gossypium hirsutum L.) & - & $8,59 \mathrm{~B}$ \\
\hline
\end{tabular}

As características das plantas de mandioca avaliadas na colheita após 18 meses do plantio, estão apresentadas na Tabela 4, como altura das plantas (cm), sobrevivência (\%), número de raízes totais e peso da cepa $(\mathrm{kg})$ que não apresentaram diferenças estatísticas entre as variedades. A variedade Engana Ladrão apresentou a maior retenção foliar $(13,73 \%)$ em comparação com as demais variedades.

As variedades Do Céu e Macaxeira Preta, tiveram maior peso total da parte aérea (1,88 e 1,75 kg por planta) em relação às Engana Ladrão e Brasília (1,14 e 1,15 kg por planta), mostrando maior acumulação de reservas que as outras na parte aérea. Considerando-se o material para forragem (material com folhas frescas) e a relação ração/ ramo total, a Macaxeira Preta e a Engana Ladrão se destacaram das demais, sendo as variedades mais promissoras para uso da parte aérea. A seleção e o uso de variedades de mandioca mais adaptadas a cada região são aspectos importantes para melhoria da rentabilidade do cultivo e redução do uso de insumos externos em qualquer parte do mundo (Ortiz, 2007). Na região Semi-Árida este aspecto é bastante relevante para os produtores pois, normalmente, os alimentos ricos em proteína são os mais ausentes nas dietas dos animais, tanto caprinos como bovinos e ovinos (Gomes \& Leal, 2006).

As variedades Engana Ladrão e Do Céu, apresentaram
Tabela 4. Características das plantas de mandioca avaliadas no momento da colheita, aos 18 meses após o plantio

\begin{tabular}{|c|c|c|c|c|}
\hline Característica avaliada & $\begin{array}{l}\text { Engana } \\
\text { Ladrão }\end{array}$ & Brasília & Do Céu & $\begin{array}{l}\text { Macaxeira } \\
\text { Preta }\end{array}$ \\
\hline Altura $(\mathrm{cm})$ & $118 A^{*}$ & $130,1 \mathrm{~A}$ & $131 \mathrm{~A}$ & $126 \mathrm{~A}$ \\
\hline $\begin{array}{l}\text { Retenção foliar } \\
\text { (\% do ramo com folhas) }\end{array}$ & $13,73 \mathrm{~A}$ & $7,42 A B$ & $6,31 \mathrm{~B}$ & $10,84 \mathrm{AB}$ \\
\hline Sobrevivência (\%) & $79 \mathrm{~A}$ & $79 \mathrm{~A}$ & $90 \mathrm{~A}$ & $85 \mathrm{~A}$ \\
\hline $\begin{array}{l}\text { Peso total de parte aérea } \\
\text { (kg por planta) }\end{array}$ & $1,14 \mathrm{~B}$ & $1,15 B$ & $1,88 \mathrm{~A}$ & $1,75 \mathrm{~A}$ \\
\hline $\begin{array}{l}\text { Peso de parte aérea para } \\
\text { forragem (g por planta) }\end{array}$ & $80 \mathrm{~A}$ & $30 \mathrm{~B}$ & $30 \mathrm{~B}$ & $100 \mathrm{~A}$ \\
\hline $\begin{array}{l}\text { Peso total de raízes } \\
\text { (kg por planta) }\end{array}$ & $2,07 \mathrm{~A}$ & $1,63 \mathrm{AB}$ & $1,84 \mathrm{~A}$ & $1,03 \mathrm{~B}$ \\
\hline Número total de raízes/planta & $6,62 \mathrm{~A}$ & 6,62 A & $5,75 \mathrm{~A}$ & $6,33 \mathrm{~A}$ \\
\hline $\begin{array}{l}\text { Peso da cepa } \\
\text { (kg por planta) }\end{array}$ & $0,19 \mathrm{~A}$ & $0,23 \mathrm{~A}$ & $0,23 \mathrm{~A}$ & $0,21 \mathrm{~A}$ \\
\hline $\begin{array}{l}\text { Produtividade de raízes } \\
\text { (kg por ha) }\end{array}$ & $26 \mathrm{~A}$ & $20 A B$ & $23 \mathrm{~A}$ & $13 \mathrm{~B}$ \\
\hline $\begin{array}{l}\text { Produtividade de parte aérea } \\
\text { (kg por ha) }\end{array}$ & $14 \mathrm{~B}$ & $14 \mathrm{~B}$ & $23 \mathrm{~A}$ & $22 \mathrm{~A}$ \\
\hline $\begin{array}{l}\text { Produção total - parte } \\
\text { aérea+raízes + cepa } \\
\text { (kg por planta) }\end{array}$ & $3,0 \mathrm{~A}$ & $3,0 \mathrm{~A}$ & $4,0 \mathrm{~A}$ & $3,0 \mathrm{~A}$ \\
\hline Índice de colheita (\%) & $69 \mathrm{~A}$ & 53 B & $46 \mathrm{C}$ & $35 \mathrm{D}$ \\
\hline $\begin{array}{l}\text { Percentagem de matéria seca } \\
\text { nas raízes }(\%)^{\star \star}\end{array}$ & $23 \mathrm{~A}$ & $21 \mathrm{~A}$ & $22 \mathrm{~A}$ & $23 \mathrm{~A}$ \\
\hline $\begin{array}{l}\text { Preferência dos produtores } \\
\text { (notas de } 0 \text { a 10) }\end{array}$ & $9 \mathrm{~A}$ & $8 \mathrm{~B}$ & $9 \mathrm{AB}$ & $5 \mathrm{C}$ \\
\hline Relação ração/ ramo total & $7,01 \mathrm{~A}$ & $2,61 \mathrm{~B}$ & $1,6 \mathrm{~B}$ & $5,71 \mathrm{~A}$ \\
\hline
\end{tabular}
pelo teste de Tukey. ** Método gravimétrico (Grossman \& Freitas, 1950)

maior peso total de raízes por planta $(2,07$ e 1,84 kg por planta). Referidos valores correspondem a uma produtividade de 26 e 23 t ha $^{-1}$ de raízes, respectivamente, sendo praticamente o dobro da média nacional nos últimos 05 anos (13 $\left.\mathrm{t} \mathrm{ha}^{-1}\right)$ e quase três vezes mais que a média do estado do Piauí (10 t ha-1) (IBGE, 2007). As variedades consideradas mansas (HCN até $100 \mathrm{mg} \mathrm{kg}^{-1}$ de polpa de raiz fresca), normalmente apresentam produtividade de raízes menores que as consideradas bravas (HCN acima de $100 \mathrm{mg} \mathrm{kg}^{-1}$ de polpa de raiz fresca (Borges et al., 2002)) porém, no presente trabalho, até as variedades mansas, como a Macaxeira Preta, mostraram produtividade acima da média regional (13 t ha $\left.{ }^{-1}\right)$, o que pode ser considerado satisfatório, haja vista que asseguram alimentação humana para os períodos secos. O índice de colheita (\%) apresentou variação de 35 a $60 \%$ entre as variedades, destacando-se a Engana Ladrão, com o maior índice (60\%); este índice de colheita demonstra que a variedade Engana Ladrão foi a mais eficiente na produção de raízes, em detrimento da parte aérea e, considerando-se sua alta retenção foliar, conclui-se que a mesma sobrevive melhor aos períodos de estiagem em comparação com as demais variedades testadas. A preferência dos produtores pela variedade Engana Ladrão coincidiu com os resultados obtidos neste trabalho e com os de outros autores (Silva et al., 2005).

A sobrevivência das plantas, o número total de raízes por planta, o peso da cepa, a produção total e a matéria seca das raízes, não diferiram estatisticamente entre as variedades testadas. As variedades Macaxeira Preta e Do Céu se 
destacaram quanto à produção de parte aérea e às Engana Ladrão e Brasília, quanto à produção de raízes, e podem ser escolhidas de acordo com a finalidade de uso na propriedade agrícola. O uso da parte aérea da mandioca é uma boa opção de alimentação do rebanho (Cavalcanti \& Araújo, 2000) e no presente trabalho as produtividades foram obtidas com o manejo de uma única colheita da mandioca, ou seja, aos 18 meses após o plantio. Caso seja usado, por exemplo, o sistema de podas sucessivas da mandioca, a produtividade de parte aérea poderá aumentar em até 30\% (Silva et al., 2005; Silva \& Santana, 2005) otimizando, assim, o uso dos recursos disponíveis. Apenas a variedade local (Macaxeira Preta), apresentou produtividade inferior às demais, sendo igual à média nacional $\left(13 \mathrm{t} \mathrm{ha}^{-1}\right)$; este resultado se deve ao sistema, como um todo, pois além da aplicação de calcário e do plantio de espécies em consórcio, as variedades escolhidas para o teste foram as que apresentaram bom crescimento em locais próximos ao do estudo; além disso, foram selecionadas manivas com bom estado fisiológico, boa quantidade de reserva de nutrientes e bom aspecto fitossanitário. As práticas utilizadas contrinbuíram para o aumento de produtividade, consolidando um sistema de produção de mandioca com maior sustentabilidade para as áreas dependentes de chuva.

\section{CONCLUSÕES}

1. Com maior produtividade de raízes, a variedade Engana Ladrão mostrou-se a mais adaptada para a região.

2. A variedade Do Céu apresentou-se promissora como opção para alimentação protéica para os animais na região.

\section{AgRADECIMENTOS}

Aos agricultores do Morro Alto (sob o comando do Ednaldo); aos colegas de trabalho (Teresinha Albuquerque), Auxiliares de Operações (Espedito Paulo) e Técnicos de Laboratório (Crisóstomo, Hélio, Emanuel, Gilberto e Adalberto) que colaboraram para que o trabalho fosse realizado.

\section{LITERATURA CITADA}

Altieri, M.; Nichols, C. Uma base agroecológica para el diseño de sistemas diversificados de cultivo en el tropico. Manejo Integrado de Pragas y Agroecologia, n.73, p.8-20. 2004.

Borges, M. F.; Fukuda, W. M. G; Rossetti, A.G. Avaliação de variedades de mandioca para consumo humano. Pesquisa Agropecuária Brasileira, v.37, n.11, p.1559-1565, 2002.

Cavalcanti, J.; Araújo, G. G. L. Parte aérea da mandioca na alimentação de ruminantes na região semi-árida. Petrolina: Embrapa Semi-Árido, 2000, 22p. Circular Técnica, n.57

Fukuda, W. M. G.; Iglesias, C. e Silva, S. de O. e. Melhoramento de mandioca. Cruz das Almas: Embrapa Mandioca e Fruticultura, 2003. Série Documentos, 104.
Gomes, J. de C.; Leal, E. C. Mandioca na alimentação animal. Raspa de raízes de mandioca e feno da parte aérea da mandioca. http://sistemasdeprodução.cnptia.embrapa.br/FontesHTML/Mandioca/mandioca_tabcosteiros/Alimentacao.htm. 16 Fev. 2006.

Grossman, J.; Freitas, A. G. Determinação do teor de matéria seca pelo método de peso específico em raízes de mandioca. Revista Agronômica, v.14, p.75-80, 1950.

IBGE - Instituto Brasileiro de Geografia e Estatística, Sidra, Tabela 99 - Rendimento médio da produção por lavoura temporária, http://www.sidra.ibge.gov.br/bda/tabela/ protabl.asp?z=t\&o=10\&i=P. 18 Abr. 2007.

Lopes, C. A.; Polidoro, J. C.; Abboud, A. C. S.; Pereira, M. B. Acumulação e exportação de nitrogênio, fósforo e potássio pela cultura da mandioca consorciada com leguminosas em sistema orgânico de produção. In: Congresso Brasileiro de Mandioca, 11, 2005, Campo Grande. Resumos...Campo Grande: Embrapa Agropecuária Oeste, 2005. CD Rom

Melo, E. S.; Santana, F. S. de; Cardoso, C. E. L. Viabilidade econômica da produção de mandioca em pequena escala. In: Congresso Brasileiro de Mandioca, 11, 2005, Campo Grande. Resumos... Campo Grande: Embrapa Agropecuária Oeste, 2005. CD Rom

Moretto, A. S.; Distel, R. A.; Didone, N. G. Decomposition and nutrient dynamic of leaf litter and roots from palatable and unpalatable grasses in a semi-arid grassland. Applied Soil Ecology, v.18, p.31-37, 2001.

Ortiz, R. Improving cassava for enhancing yield, minimizing pest losses and creating wealth in Sub-Saharan Africa. In: International meeting on cassava breeding, Biotechnology and Ecology, 1, 2006, Brasília, Proceedings...Brasília: UNB, 2007. p.129-141

Otsubo. A. A; Lorenzi, J. O. Cultivo da mandioca na região Centro-Sul do Brasil. 2002. Dourados: Embrapa Agropecuária Oeste, 2004. p.51. Sistemas de Produção. 6

Perin, A.; Lima, E. A. de; Espíndola, J. A. A.; Guerra, J. G. M.; Teixeira, M. G.; Busquet, R. N. B. Contribuição da cobertura viva de solo com leguminosas herbáceas perenes no $2^{\circ}$ ciclo de produção de bananeiras cultivar nanicão. Seropédica: Embrapa Agrobiologia, 2002. 4p. Comunicado Técnico 53

Perpétuo, I. F. Norte e Nordeste usam técnicas pré-cabralinas. http://www1.folha.uol.com.br/fol/brasil50 00/comida4.htm.. 02 Ago. 2006.

Putthacharoen, S.; Howler, R. H.; Jantarwat, S.; Vichukit, V. Nutrient uptake and soil erosion losses in cassava and six other crops in a Psament in eastern Thailand. Field Crops Research, v.57, p.113-126, 1998.

Ribas, R. T.; Junqueira, R. M.; Oliveira, F. L. de; Guerra, J. G. M.; Almeida, D. L. de; Ribeiro, R. de L. D. Manejo da adubação verde com o quiabeiro sob manejo orgânico. Seropédica: Embrapa Agrobiologia, 2003. 4p. Comunicado Técnico, 59

Silva, A. F.; Santana, L. M. de. Crescimento de mandioca, maniçoba e pornunça conduzidas sob podas em épocas distintas na região Semi-Árida. In: Congresso Brasileiro de Mandioca, 11, 2005, Campo Grande. Resumos...Campo Grande: Embrapa Agropecuária Oeste, 2005. CD Rom 
Silva, A. F.; Santana, L. M. de; Silva, M. M. da. Comportamento de variedades de mandioca no semi-árido sob diferentes sistemas de poda. In: Congresso Brasileiro de Mandioca, 11, 2005, Campo Grande. Resumos...Campo Grande: Embrapa Agropecuária Oeste, 2005. CD Rom
Toro, J. C.; Cañas, A. Determinacion de materia seca de las raices frecas por el metodo de la gravidad especifica. In: Ospina, B.; Ceballos, H. La yuca em tercer milenio. Sistemas modernos de produción, processamiento, utilización y comercialización. Cali: CIAT. 1.ed. 2002. 7p. 